\title{
To divert or not to divert prehospital ST-elevation myocardial infarction: What is the question?
}

\author{
Jerome R. Hoffman, MA, MD; Richelle J. Cooper, MD, MSHS
}

See related articles on pages 473 and 481

Version française À la page 426

$\mathrm{T}$ ime is myocardium" ... we all know that. Or do we? Outcomes are far better with thrombolysis in the first hour of symptoms than they are an hour later. So in the patient with hyperacute symptoms, time is indeed myocardium. ${ }^{1,2}$ But there's virtually no difference between treatment at 4 hours versus 6 hours, so ... perhaps time is not always myocardium. In addition, since percutaneous coronary intervention (PCI), even when delayed up to 2 hours, is better than a thrombolytic for the average ST-elevation myocardial infarction (STEMI) patient, it is apparent that other factors can be more important than time to treatment. ${ }^{3}$

This issue of CJEM includes 2 well-written articles ${ }^{4,5}$ that address the role of prehospital providers in the rapid identification and treatment of possible myocardial ischemia. This is ostensibly because emergency medical services (EMS) can impact both the time to treatment (with rapid diagnosis, the notification of the receiving centre and even, perhaps, with immediate administration of a thrombolytic) and the availability of optimal treatment (with directed transport to a centre where PCI is available). Although both studies appear internally valid, they come to strikingly opposite conclusions about what EMS providers should be doing. How did this happen, and which, if either, is "correct"?

Schull and colleagues ${ }^{4}$ examine several prehospital practices in 5 Canadian provinces and report what they consider to be underuse of systematic strategies to reduce time to reperfusion. ${ }^{4}$ It is critical to understand that although the results of their study are unimpeachable in that few EMS systems have undertaken the strategies they recommend, the conclusions are only as good as the investigators' basic assumptions: that these strategies are not only proven to be beneficial, but that the benefits from their implementation will outweigh the costs, both economic and medical. We have no problem agreeing with the former: there is strong evidence that the strategies they recommend do provide at least some benefit. We believe the latter ("such benefit justifies the cost") is more problematic.

If one starts with the belief that these strategies should be implemented, it is impossible to argue with the conclusion that the current state of EMS is sadly lacking. In contrast, Brooks and coworkers ${ }^{5}$ start by challenging the concept that these strategies should be implemented; in fact they go further, questioning the notion that direct transfer to a PCI centre is proven to be better than the early use of thrombolytics. To address this, they performed a systematic review and meta-analysis, and, contrary to Schull and colleagues, conclude that prehospital direct transport to a PCI centre is not proven to be beneficial. They base this on summary results that fail to find a difference between the 2 approaches that is "statistically significant," which is the same as saying that the degree of difference found could be due to chance alone. Without addressing the authors' inclusion of heterogeneous data, not all of which comes from randomized controlled trials, or the evidence from excluded nonEMS studies that strongly favours PCI over lytics, or even the notion that $p<0.05$ represents a scientific yes or no cut-point rather than simply convention, we should note that their own point estimate clearly favours PCI. A relative risk (RR) of 0.51 means that a bad outcome is only about half as likely with PCI. Even though it is possible that if we studied thousands more patients we'd find that the true RR is higher, or even 1 (i.e., there

From the David Geffen School of Medicine at the University of California at Los Angeles, Los Angeles, Calif.

All editorial matter represents the views of the authors and not necessarily those of CJEM or the Canadian Association of Emergency Physicians.

CJEM 2009;11(5):423-5 
is no difference), the most likely $\mathrm{RR}$, based on the available data, is 0.51 ; and the lower confidence interval boundary suggests it could even be far less!

Whenever a study provides a point estimate that, if verified with more data, would be clinically important, even while it is not yet statistically significant, we should immediately understand that the available information is, by definition, underpowered to find an important difference. It is reasonable to think that the calculated difference might be due to chance alone (although $p=$ 0.06 is, of course, essentially identical to a "significant" difference of $p=0.05$ and the latter case is typically considered proven!) and reject the idea that the point estimate would automatically stay the same with more patients in the study. But we should similarly reject the notion that " $p>0.05$ " means "not real" (i.e., failure to "prove" benefit is enormously different than proof of the absence of benefit).

Two of the authors in the Brooks and coworkers study disclose ties to drug companies that sell thrombolytics. Such disclosure can only have value if we, the readers, are willing to think about the potential impact of the relationship being disclosed. Thus, although we have no wish to speculate about whether the analysis was influenced by this relationship, we feel it is fair to note that the companies involved will undoubtedly be pleased with the written conclusions.

We believe that there is more than adequate information to reject the conclusion of "no difference" between PCI and thrombolytics, and therefore agree that the assumptions made by Schull and colleagues are in some sense correct. However, given that, we would suggest that the relevant question is not "Why aren't we doing this?" but rather "Is it worth doing?" For this we must consider not only the probable magnitude of the benefit, but also the known and possible financial and medical harm that would come from implementing such a strategy.

A formal cost-effectiveness analysis is complex and requires a good deal of information that, as noted by Brooks and coworkers, is currently unknown. Furthermore, even the best such analysis always relies on assumptions that may not be entirely justified. Nevertheless, a recent decision analysis with conservative assumptions by Wang and coworkers ${ }^{6}$ concluded that, in terms of patient outcome, the small benefit that would arise from structured EMS diversion policies would come at a cost that seems hard to justify.

It is important to consider the possible clinical harms related to reconfiguring EMS systems to allow for rapid diversion to PCI. When an ambulance is unavailable because it is diverted, how many other patients may suffer harm? When an ambulance believes the most important aspect of its response is a sense of time urgency, how many lights-and-sirens accidents will it take to wipe out any potential gain? When the diagnosis is presumed in the field, and the emergency physician (EP) is explicitly or implicitly removed from the equation, how many under- or overtreatments of different, or concomitant, disease processes (such as aortic dissection) will it take to change the medical equation from benefit to harm? In that regard, we believe the specialty of emergency medicine is fundamentally grounded in the concept that the EP, rather than simply a triage doctor, is the single most critical element in maximizing outcomes for all our patients.

With all this in mind, we propose the following thought experiment. We don't pretend that our numbers are even remotely precise (for a handful of reasons, precise estimates are not currently possible), but believe that the conclusions to which they seem to lead would remain reasonable even if we are off by a factor of 10 or more: imagine that for every 100000 chest pain patients, one-half are seriously considered as having possible acute coronary syndrome, and of these $50000 \mathrm{pa}-$ tients (5\%) actually have an acute myocardial infarction (AMI). If 1 in 5 of these 2500 AMI patients has a true STEMI, we're down to 500 STEMI patients from the original 100000 . Now let's use available literature to assume that the benefit of PCI over thrombolytics is an absolute risk reduction for death of $1 \%$; so changing the EMS system to ensure that every chest pain patient has a chance to be taken directly to PCI, compared with an approach in which none of them would get PCI (but all might get a thrombolytic if appropriate) would be expected to save 5 extra lives from our original cohort of 100 000. Since our current system brings at least some patients directly to PCI, the number of expected "saves" would actually be less; and this is comparing PCI with thrombolysis, rather than comparing immediate transport to PCI with slightly delayed PCI, in which case there may be essentially no benefit at all!

This takes us back to time and myocardium. If EMS continues to transport patients to an appropriate ED with an appropriate EP, that physician can decide whether the electrocardiogram (ECG) shows a STEMI in the first place. If the answer is yes, the EP can then consider whether special circumstances require a modification of the standard approach ... because the ECG changes are actually because of aortic dissection, or because the myocardial infarction was caused by 
cocaine, or because of any number of other important clinical considerations for which EPs are trained. Then that EP, if he or she is working in a facility without PCI, could decide to transfer the very few patients who might benefit from PCI, at a financial cost that would almost certainly be trivial compared with the cost of revamping all of EMS, and at a medical cost of perhaps an hour's delay; a delay that is unlikely to have major consequences unless the symptoms are hyperacute. As to that rare patient who not only has a STEMI, and has come to a centre without PCI, but is also in the first hour or so of symptoms, this is precisely the circumstance where thrombolysis is extremely effective: so much so that it is unlikely that PCI offers much if any benefit, and that knowledgeable EP might appropriately decide not to transfer that particular patient at all!

Both papers in this issue of CJEM add to our knowledge of this complex issue, and each provides useful data for generating further hypotheses. We believe that current evidence is adequate, however, to conclude that although shorter time to reperfusion is generally preferable, it is of major importance in only a small subset of patients. Similarly, we agree with Schull and colleagues that current evidence supports PCI over thrombolytics. But again, only to a small degree, and in a small number of chest pain patients. Unless and until there is substantially new and different evidence, the appropriate question for EMS and for emergency medicine is whether the marginal benefit likely to accrue from primary diversion can possibly justify the likely costs and potential harms of this fundamental overhaul of our current EMS approach to patients with chest pain. We suggest that a better strategy would emphasize rapid ED assessment of all such patients, with secondary transfer for the very small subset who are likely to benefit from PCI. ${ }^{7,8}$
Competing interests: None declared.

Keywords: STEMI, prehospital diversion, PCI centre

\section{REFERENCES}

1. Boersma E, Mass AC, Deckers JW, et al. Early thrombolytic treatment in acute myocardial infarction: reappraisal of the golden hour. Lancet 1996;348:771-5.

2. Steg PG, Bonnefoy E, Chabaud S, et al.; Comparison of angioplasty and prehospital thrombolysis in acute myocardial infarction (CAPTIM) investigators. Impact of time to treatment on mortality after prehospital fibrinolysis or primary angioplasty. Circulation 2003;108:2851-6.

3. Keeley EC, Boura JA, Grines CL. Primary angioplasty versus intravenous thrombolytic therapy for acute myocardial infarction: a quantitative review of 23 randomised trials. Lancet 2003;361:13-20.

4. Schull MJ, Vaillancourt S, Donovan L, et al. Underuse of prehospital strategies to reduce time to reperfusion for ST-elevation myocardial infarction patients in 5 Canadian provinces. CJEM 2009; 5: 473-80.

5. Brooks SC, Allan KS, Welsford M. Prehospital triage and direct transport of patients with ST-elevation myocardial infarction to primary percutaneous coronary intervention centres: a systematic review and meta-analysis. CJEM 2009; 5:481-92.

6. Wang HE, Marroquin OC, Smith KJ. Direct paramedic transport of acute myocardial infarction patients to percutaneous coronary intervention centers: a decision analysis. Ann Emerg Med 2009;53:233-40.

7. De Luca G, Biondi-Zoccai G, Marino P. Transferring patients with ST-segment elevation myocardial infarction for mechanical reperfusion: a meta-regression analysis of randomized trials. Ann Emerg Med 2008;52:665-76.

8. Dalby M, Bouzamondo A, Lechat P, et al. Transfer for primary angioplasty versus immediate thrombolysis in acute myocardial infarction: a meta-analysis. Circulation 2003;108: $1809-14$

Correspondence to: Dr. Richelle J. Cooper, 924 Westwood Blvd., Suite 300, Los Angeles CA 90024; richelle@ucla.edu

\section{Erratum}

In the May 2009 issue of CJEM, the institutional affiliation for CAEP abstracts 12, 21, 73, 78, 100 and 119 was listed incorrrectly. The correct institution is WakeMed Health and Hospitals, Raleigh, NC. 